\title{
Asteroid Hovering Missions from a Fuel-Consumption Viewpoint
}

\author{
Wei Wang* Di Wu ${ }^{\dagger}$ \\ School of Aerospace Engineering, Tsinghua University, 100084 Beijing, \\ People's Republic of China \\ Giovanni Mengali; Alessandro A. Quarta ${ }^{\S}$ \\ Department of Civil and Industrial Engineering, University of Pisa, I-56122 Pisa, Italy \\ and \\ Hexi Baoyin $\mathbb{1}$ \\ School of Aerospace Engineering, Tsinghua University, 100084 Beijing, \\ People's Republic of China
}

\section{Introduction}

Over the last decades, small celestial bodies like asteroids have drawn a growing interest due to their importance not only for scientific explorations, but also for practical applications $[1,2]$. The value of asteroid detection is clearly confirmed by some past mission tasks, such as NEAR-Shoemaker [3], characterized by a rendezvous with 433 Eros, and Hayabusa [4], the first successful sample return prober with its target at 25143 Itokawa.

Different ways exist to plan missions for asteroid exploration, for example, by inserting a spacecraft into natural periodic orbits [5], exploiting high-energy non-Keplerian orbits [6,7], or using stationary hovering orbits [8]. In particular, the idea of placing the hovering spacecraft at some artificial equilibrium points [8-10] of the rotating asteroid is considered one of the best ways to acquire an area image of special interest within a certain field of vision [1], useful for identifying candidate sites before landing (or impacting) on the asteroid's surface [11].

So far, much effort has been devoted to the development of closed-loop hovering control strategies [12], aimed at tracking a prescribed trajectory even in the presence of a complex gravitational field. In this regard, a robust performance is usually the primary goal for many advanced control paradigms [13-15]. The effectiveness of those methods is in their capability of accounting for uncertain dynamical environments and other realistic factors such as the existence of dead-band [16] in the control system. Some open questions, however, still exist, which deserve a further study. For instance, in the available literature, the asteroid hovering problem is seldom addressed in terms of propellant mass necessary to guarantee a certain mission length. However, because the asteroids' shape is in general nonspherical and even highly irregular, the overall fuel cost necessary to counterbalance the asteroid gravity is strongly dependent on the hovering position, especially for those missions requiring sustained close-proximity operations. This observation necessitates a clear insight in identifying a direct relationship between the hovering position and the propellant consumption. In fact, if some flexibility is allowed in the choice of hovering point, such a degree of freedom may be exploited to optimize the total fuel consumption, thus providing more redundancy for other mission tasks, such as a deep impact or a soft landing on the asteroid.

The aim of this Note is to provide a comprehensive study of asteroid hovering from a fuel-consumption viewpoint, under the assumption that the asteroid is modelled as a second-degree and a second-order non-spherical gravitational field. Our analysis first relies on an analytical, although approximate, approach to determine the critical hovering orientations and to quantify the fuel-consumption extrema. The linear stability for a given hovering distance is investigated, and a homotopic method is adopted as an effective tool to emphasize the fuel-optimal control problem. In particular, optimal trajectories toward a stable, minimum-fuel, hovering point are found by first solving the simpler energy-optimal problem. The initial (unknown) costate vector is calculated with a normalization technique, in such a way that the sensitivity of the two-point boundary-value problem (TPBVP) to the initial guess is significantly mitigated.

The Note is organized as follows. Section II discusses the necessary conditions for asteroid hovering. Section III investigates the fuel-consumption extrema and the corresponding critical hovering orientations using an analytical point of view. In Section IV, the linear stability is analyzed with a numerical approach. In Section V, a homotopic method, in association with a costate vector normalization technique, is adopted to study the trajectory optimization problem. Finally, some concluding remarks are proposed.

\section{Mathematical Preliminaries}

Consider an asteroid of uniform density that rotates at a constant rate about its maximum axis of inertia and a spacecraft, propelled by a continuous thrust propulsion system, which moves subject to the asteroid gravitational field; see Fig. 1. The dynamic model describing the spacecraft motion is formulated in a body-fixed (rotating) reference frame $\mathcal{T}(O ; \hat{\boldsymbol{x}}, \hat{\boldsymbol{y}}, \hat{\boldsymbol{z}})$, whose origin $O$ coincides with the asteroid center-of-mass, while axes $\{\hat{\boldsymbol{x}}, \hat{\boldsymbol{y}}, \hat{\boldsymbol{z}}\}$ are aligned with the minimum, intermediate, and maximum axis of inertia of the asteroid, respectively. In this model, the asteroid spin axis is assumed to coincide with the $\hat{\boldsymbol{z}}$-axis, that is, the angular velocity vector is $\boldsymbol{\omega} \equiv \omega \hat{\boldsymbol{z}}$, where $\omega$ is the constant asteroid spin rate.

The spacecraft dynamics is described by the second-order differential equation [17]

$$
\ddot{\boldsymbol{r}}+2 \boldsymbol{\omega} \times \dot{\boldsymbol{r}}+\boldsymbol{\omega} \times(\boldsymbol{\omega} \times \boldsymbol{r})=-\frac{\partial U}{\partial \boldsymbol{r}}+\boldsymbol{u}
$$

\footnotetext{
*Research Assistant, wei_wang@mail.tsinghua.edu.cn.

${ }^{\dagger}$ Ph.D. Candidate, wud17@mails.tsinghua.edu.cn.

${ }_{\ddagger}^{\ddagger}$ Professor, g.mengali@ing.unipi.it. Senior Member AIAA.

${ }^{\S}$ Professor, a.quarta@ing.unipi.it. Associate Fellow AIAA

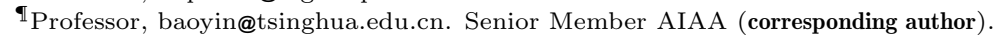




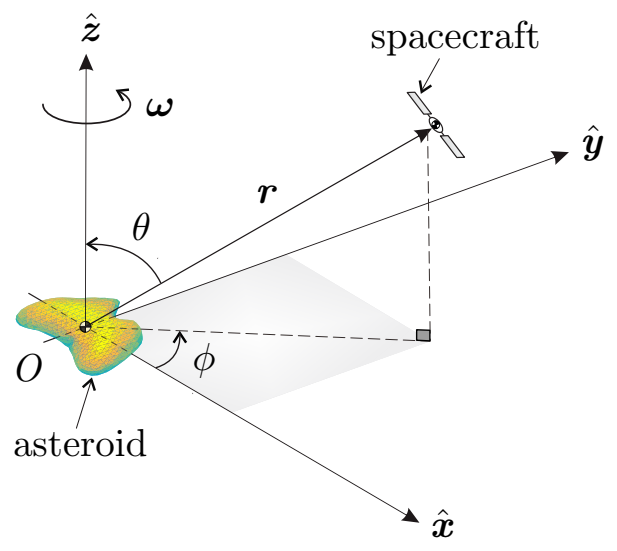

Figure 1 Rotating reference frame and spacecraft relative position.

where $\boldsymbol{r} \triangleq[x, y, z]^{\mathrm{T}}$ is the spacecraft position vector, $\boldsymbol{u}$ is the propulsive acceleration vector, and $U$ is the asteroid gravitational potential function. Assuming a second-degree and second-order asteroid gravitational potential [1], the potential function can be written as

$$
U=-\frac{\mu}{r}+\frac{\mu}{2 r^{5}} \widetilde{C}_{20}\left(x^{2}+y^{2}-2 z^{2}\right)-\frac{3 \mu}{r^{5}} \widetilde{C}_{22}\left(x^{2}-y^{2}\right)
$$

where $r \triangleq\|\boldsymbol{r}\|$ is the asteroid-spacecraft distance, $\mu$ is the asteroid gravitational parameter. The augmented Stokes coefficients $\left\{\widetilde{C}_{20}, \widetilde{C}_{22}\right\}$ are defined as $\widetilde{C}_{20} \triangleq R_{0}^{2} C_{20}$ and $\widetilde{C}_{22} \triangleq R_{0}^{2} C_{22}$, in which $\left\{C_{20}, C_{22}\right\}$ are the second-degree and second-order gravity field harmonic (Stokes) coefficients, and $R_{0}$ is the normalizing distance.

For convenience, introduce the dimensionless (positive) parameter $\kappa \triangleq \omega^{2} /\left(\mu / r^{3}\right)$ and a set of spherical coordinates $\{r, \theta, \phi\}$, defined as $x \triangleq r \sin \theta \cos \phi, y \triangleq r \sin \theta \sin \phi, z \triangleq r \cos \theta$, where $\theta \in[0, \pi]$ rad is the angle between the $\hat{\boldsymbol{z}}$-axis and the position vector $\boldsymbol{r}$, while $\phi \in[0,2 \pi]$ rad is the angle between the $\hat{\boldsymbol{x}}$-axis and the projection of $\boldsymbol{r}$ onto the $(\hat{\boldsymbol{x}}, \hat{\boldsymbol{y}})$ plane; see Fig. 1 . An asteroid hovering condition is obtained by placing the spacecraft at some artificial equilibrium point in the frame $\mathcal{T}$, which amounts to enforcing the condition $\dot{\boldsymbol{r}}=\ddot{\boldsymbol{r}}=\mathbf{0}$ in Eq. (1). Accordingly, the required spacecraft propulsive acceleration is

$$
\boldsymbol{u}=\boldsymbol{\omega} \times(\boldsymbol{\omega} \times \boldsymbol{r})+\frac{\partial U}{\partial \boldsymbol{r}}
$$

Note that the expression asteroid hovering here refers to a body-fixed hovering [9], whereas an inertial hovering [18] (which is useful for remote sensing, imaging, and surface mapping) is beyond the scope of this Note. Using Eq. (3), the components of the propulsive acceleration vector are given by

$$
\begin{aligned}
& u_{x}=\frac{\mu}{r^{2}} \sin \theta \cos \phi\left\{(1-\kappa)+\frac{\widetilde{C}_{20}}{r^{2}}\left[1-\frac{5}{2}\left(\sin ^{2} \theta-2 \cos ^{2} \theta\right)\right]-\frac{\widetilde{C}_{22}}{r^{2}}\left[6-15 \sin ^{2} \theta\left(\cos ^{2} \phi-\sin ^{2} \phi\right)\right]\right\} \\
& u_{y}=\frac{\mu}{r^{2}} \sin \theta \sin \phi\left\{(1-\kappa)+\frac{\widetilde{C}_{20}}{r^{2}}\left[1-\frac{5}{2}\left(\sin ^{2} \theta-2 \cos ^{2} \theta\right)\right]+\frac{\widetilde{C}_{22}}{r^{2}}\left[6+15 \sin ^{2} \theta\left(\cos ^{2} \phi-\sin ^{2} \phi\right)\right]\right\} \\
& u_{z}=\frac{\mu}{r^{2}} \cos \theta\left\{1-\frac{\widetilde{C}_{20}}{r^{2}}\left[2+\frac{5}{2}\left(\sin ^{2} \theta-2 \cos ^{2} \theta\right)\right]+\frac{\widetilde{C}_{22}}{r^{2}} 15 \sin ^{2} \theta\left(\cos ^{2} \phi-\sin ^{2} \phi\right)\right\}
\end{aligned}
$$

which are functions of the triplet $\{\kappa, \theta, \phi\}$. Note that, as a consequence of the assumption of uniformly rotating asteroid, the vector $\boldsymbol{u}$ is time-invariant.

\section{Fuel-Consumption Extrema}

The total fuel consumption is a crucial point for long-term hovering missions, because the spacecraft has to compensate for the asteroid local gravity through a continuous thrust. In this section, starting from Eqs. (4)-(6) and assigning the asteroid-spacecraft distance, the extrema of fuel consumption for hovering control will be analytically obtained along with the corresponding critical spacecraft directions.

\section{Critical Hovering Directions}

The fuel consumption can be analyzed by studying the spatial variation of the square of the propulsive acceleration magnitude, that is, $\|\boldsymbol{u}\|^{2}$. For a given hovering distance $\rho$, the extrema of $\|\boldsymbol{u}\|^{2}$ can be obtained by solving a static optimization problem with equality constraints in the form [19]

$$
\left\{\theta^{\star}, \phi^{\star}\right\}=\arg \min _{\{\theta, \phi\}}\|\boldsymbol{u}\|^{2} \quad \text { s.t. } \quad\left\|\boldsymbol{r}\left(\theta^{\star}, \phi^{\star}\right)\right\|=\rho
$$

where $\left\{\theta^{\star}, \phi^{\star}\right\}$ are the angles that give the (critical) hovering directions from a fuel-consumption viewpoint. The augmented cost functional $\Phi$ is

$$
\Phi=\|\boldsymbol{u}\|^{2}+\nu\left(\|\boldsymbol{r}\|^{2}-\rho^{2}\right)
$$


where $\nu$ is a Lagrange multiplier. The necessary conditions for an extremum are

$$
\frac{\partial \Phi}{\partial \theta}=0, \quad \frac{\partial \Phi}{\partial \phi}=0 \quad \text { s.t. } \quad\|\boldsymbol{r}\|=\rho
$$

Equations (9) constitute a system of three algebraic equations in the three variables $\{\theta, \phi, \nu\}$, of which an analytical solution is difficult to find when the actual expressions of the components $\left\{u_{x}, u_{y}, u_{z}\right\}$, given by Eqs. (4)-(6), are substituted into Eq. (8). An approximate expression of $\|\boldsymbol{u}\|^{2} \equiv\left(u_{x}^{2}+u_{y}^{2}+u_{z}^{2}\right)$ may however be derived by observing that the augmented harmonics coefficients $\left\{\widetilde{C}_{20}, \widetilde{C}_{22}\right\}$ are small compared to $r^{2}$, viz.

$$
\left|\frac{\widetilde{C}_{20}}{r^{2}}\right| \ll 1, \quad\left|\frac{\widetilde{C}_{22}}{r^{2}}\right| \ll 1, \quad\left|\frac{\widetilde{C}_{20}}{r^{2}}\right| \ll|\kappa-1|, \quad\left|\frac{\widetilde{C}_{22}}{r^{2}}\right| \ll|\kappa-1|
$$

Since $\kappa$ is usually not close to 1, the last two assumptions in Eq. (10) naturally hold true in most cases. The first-order approximation of $\|\boldsymbol{u}\|^{2}$ is obtained from Eqs. (4)-(6) by neglecting the terms including $\left\{\widetilde{C}_{20}^{2} / r^{4}, \widetilde{C}_{22}^{2} / r^{4}, \widetilde{C}_{20} \widetilde{C}_{22} / r^{4}\right\}$. After some algebraic manipulations, the result turns out to be

$$
\begin{aligned}
\|\boldsymbol{u}\|^{2} & \simeq \frac{\mu^{2}}{r^{4}}\left\{1+\frac{6 \widetilde{C}_{20}}{r^{2}}+\frac{15 \kappa \widetilde{C}_{20}-30 \kappa \widetilde{C}_{22} \cos 2 \phi}{r^{2}} \sin ^{4} \theta\right. \\
& \left.+\left[\kappa^{2}-2 \kappa+\frac{6(2 \kappa+3) \widetilde{C}_{22} \cos 2 \phi-3(4 \kappa+3) \widetilde{C}_{20}}{r^{2}}\right] \sin ^{2} \theta\right\}
\end{aligned}
$$

Substituting Eq. (11) into Eq. (8), the necessary conditions (9) become

$$
\begin{aligned}
& \sin 2 \theta(\mathcal{A} \cos 2 \theta+\mathcal{B})=0 \\
& \sin ^{2} \theta \sin 2 \phi\left[5 \kappa \sin ^{2} \theta-(2 \kappa+3)\right]=0
\end{aligned}
$$

where $\mathcal{A}$ and $\mathcal{B}$ are two auxiliary functions, defined as

$$
\begin{aligned}
& \mathcal{A}=15 \kappa\left(2 \widetilde{C}_{22} \cos 2 \phi-\widetilde{C}_{20}\right) \\
& \mathcal{B}=\left(\kappa^{2}-2 \kappa\right) \rho^{2}+(3 \kappa-9) \widetilde{C}_{20}+18(1-\kappa) \widetilde{C}_{22} \cos 2 \phi
\end{aligned}
$$

where $\rho=\sqrt[3]{\mu \kappa / \omega^{2}}$. Thus, Eqs. (14)-(15) are functions of $\{\phi, \kappa\}$, so that Eqs. (12)-(13) are nonlinear functions of $\{\theta, \phi, \kappa\}$. For a given value of $\kappa$, the solution set of Eq. (12) is

$$
\mathcal{S}_{1}:\left\{\begin{array}{lll}
\theta=\{0, \pi / 2, \pi\} & \cap & \forall \phi \in[0,2 \pi] \\
\mathcal{A} \cos 2 \theta+\mathcal{B}=0 & \cap & |\mathcal{A} / \mathcal{B}| \geq 1
\end{array}\right.
$$

while that of Eq. (13) is

$$
\mathcal{S}_{2}:\left\{\begin{array}{lll}
\theta=\left\{0, \pi, \arcsin \sqrt{\frac{2 \kappa+3}{5 \kappa}}, \pi-\arcsin \sqrt{\left.\frac{2 \kappa+3}{5 \kappa}\right\}}\right. & \cap & \forall \phi \in[0,2 \pi] \\
\phi=\{0, \pi / 2, \pi, 3 \pi / 2,2 \pi\} & \cap \quad \forall \theta \in[0, \pi]
\end{array}\right.
$$


The critical hovering directions $\left\{\theta^{\star}, \phi^{\star}\right\}$ correspond to when Eqs. (16)-(17) are satisfied simultaneously, that is, $\left\{\theta^{\star}, \phi^{\star}\right\} \in$ $\mathcal{S}_{1} \cap \mathcal{S}_{2}$. Therefore, the possible solution sets $\left\{\mathcal{S}_{a}, \mathcal{S}_{b}, \ldots, \mathcal{S}_{e}\right\}$ can be written as

$$
\begin{aligned}
& \mathcal{S}_{a}: \quad \theta^{\star}=\{0, \pi\} \quad \cap \quad \forall \phi^{\star} \in[0,2 \pi] \\
& \mathcal{S}_{b}: \quad \theta^{\star}=\frac{\pi}{2} \quad \cap \quad \phi^{\star}=\{0, \pi / 2, \pi, 3 \pi / 2,2 \pi\} \\
& \mathcal{S}_{c}: \quad \Delta_{1} \geq 0 \quad \cap \quad \theta^{\star}=\left\{\arcsin \sqrt{\frac{2 \kappa+3}{5 \kappa}}, \pi-\arcsin \sqrt{\frac{2 \kappa+3}{5 \kappa}}\right\} \\
& \cap \quad \phi^{\star}=\left\{\frac{1}{2} \arccos \frac{9 \widetilde{C}_{20}+\left(\kappa^{2}-2 \kappa\right) \rho^{2}}{6 \widetilde{C}_{22}(2 \kappa+3)}, \pi-\frac{1}{2} \arccos \frac{9 \widetilde{C}_{20}+\left(\kappa^{2}-2 \kappa\right) \rho^{2}}{6 \widetilde{C}_{22}(2 \kappa+3)},\right. \\
& \left.\pi+\frac{1}{2} \arccos \frac{9 \widetilde{C}_{20}+\left(\kappa^{2}-2 \kappa\right) \rho^{2}}{6 \widetilde{C}_{22}(2 \kappa+3)}, 2 \pi-\frac{1}{2} \arccos \frac{9 \widetilde{C}_{20}+\left(\kappa^{2}-2 \kappa\right) \rho^{2}}{6 \widetilde{C}_{22}(2 \kappa+3)}\right\} \\
& \mathcal{S}_{d}: \quad \Delta_{2} \geq 0 \quad \cap \quad \theta^{\star}=\left\{\begin{array}{l}
\frac{1}{2} \arccos \frac{\left(\kappa^{2}-2 \kappa\right) \rho^{2}+(3 \kappa-9) \widetilde{C}_{20}+18(1-\kappa) \widetilde{C}_{22}}{15 \kappa\left(\widetilde{C}_{20}-2 \widetilde{C}_{22}\right)},
\end{array}\right. \\
& \left.\pi-\frac{1}{2} \arccos \frac{\left(\kappa^{2}-2 \kappa\right) \rho^{2}+(3 \kappa-9) \widetilde{C}_{20}+18(1-\kappa) \widetilde{C}_{22}}{15 \kappa\left(\widetilde{C}_{20}-2 \widetilde{C}_{22}\right)}\right\} \cap \quad \phi^{\star}=\{0 ; \pi ; 2 \pi\} \\
& \mathcal{S}_{e}: \quad \Delta_{3} \geq 0 \quad \cap \quad \theta^{\star}=\left\{\begin{array}{l}
\frac{1}{2} \arccos \frac{\left(\kappa^{2}-2 \kappa\right) \rho^{2}+(3 \kappa-9) \widetilde{C}_{20}-18(1-\kappa) \widetilde{C}_{22}}{15 \kappa\left(\widetilde{C}_{20}+2 \widetilde{C}_{22}\right)},
\end{array}\right. \\
& \left.\pi-\frac{1}{2} \arccos \frac{\left(\kappa^{2}-2 \kappa\right) \rho^{2}+(3 \kappa-9) \widetilde{C}_{20}-18(1-\kappa) \widetilde{C}_{22}}{15 \kappa\left(\widetilde{C}_{20}+2 \widetilde{C}_{22}\right)}\right\} \quad \cap \quad \phi^{\star}=\left\{\frac{\pi}{2} ; \frac{3 \pi}{2}\right\}
\end{aligned}
$$

where the discriminants $\Delta_{1}, \Delta_{2}$, and $\Delta_{3}$ are given by

$$
\begin{aligned}
& \Delta_{1}=6 \widetilde{C}_{22}(2 \kappa+3)-\left|9 \widetilde{C}_{20}+\left(\kappa^{2}-2 \kappa\right) \rho^{2}\right| \\
& \Delta_{2}=\left|15 \kappa\left(2 \widetilde{C}_{22}-\widetilde{C}_{20}\right)\right|-\left|\left(\kappa^{2}-2 \kappa\right) \rho^{2}+(3 \kappa-9) \widetilde{C}_{20}+18(1-\kappa) \widetilde{C}_{22}\right| \\
& \Delta_{3}=\left|15 \kappa\left(2 \widetilde{C}_{22}+\widetilde{C}_{20}\right)\right|-\left|\left(\kappa^{2}-2 \kappa\right) \rho^{2}+(3 \kappa-9) \widetilde{C}_{20}-18(1-\kappa) \widetilde{C}_{22}\right|
\end{aligned}
$$

According to Eqs. (18)-(22), $\mathcal{S}_{a}$ and $\mathcal{S}_{b}$ are solution sets of Eqs. (12)-(13) in all cases, whereas $\mathcal{S}_{c}, \mathcal{S}_{d}$, and $\mathcal{S}_{e}$ are conditional sets, depending on the sign of $\Delta_{1}, \Delta_{2}$, and $\Delta_{3}$. Note that, in order to identify the minima of $\|\boldsymbol{u}\|^{2}$, the solutions (18)-(22) must be further analyzed by examining the sign of the second-order (or high order if saddle points appear) partial derivative at the critical hovering directions $\left\{\theta^{\star}, \phi^{\star}\right\}$.

Consider, for example, asteroid 433 Eros, of which the main physical parameters [13] are reported in Tab. 1. The solution

Table 1 Physical Parameters of asteroid 433 Eros [13].

\begin{tabular}{cc}
\hline \hline parameter & value \\
\hline$\mu$ & $4.462 \times 10^{-4} \mathrm{~km}^{3} / \mathrm{s}^{2}$ \\
$\omega$ & $3.31 \times 10^{-4} \mathrm{rad} / \mathrm{s}$ \\
$\widetilde{C}_{20}$ & $-26.755 \mathrm{~km}^{2}$ \\
$\widetilde{C}_{22}$ & $12.752 \mathrm{~km}^{2}$ \\
\hline \hline
\end{tabular}

set $\mathcal{S}$ of all critical hovering directions is written in terms of pairs $\left\{\theta^{\star}, \phi^{\star}\right\}$ as

$$
\mathcal{S}=\left\{\begin{array}{lll}
\mathcal{S}_{a} \cup \mathcal{S}_{b} \cup \mathcal{S}_{d}: & \Delta_{1}<0 \cap \Delta_{2}>0 \cap \Delta_{3} \leq 0 & \text { if } \quad \kappa \in\left(1, \kappa_{1}^{\star}\right] \\
\mathcal{S}_{a} \cup \mathcal{S}_{b} \cup \mathcal{S}_{d} \cup \mathcal{S}_{e}: & \Delta_{1} \leq 0 \cap \Delta_{2}>0 \cap \Delta_{3}>0 & \text { if } \quad \kappa \in\left(\kappa_{1}^{\star}, \kappa_{2}^{\star}\right] \\
\mathcal{S}_{a} \cup \mathcal{S}_{b} \cup \mathcal{S}_{c} \cup \mathcal{S}_{d} \cup \mathcal{S}_{e}: & \Delta_{1}>0 \cap \Delta_{2}>0 \cap \Delta_{3} \geq 0 & \text { if } \quad \kappa \in\left(\kappa_{2}^{\star}, \kappa_{3}^{\star}\right] \\
\mathcal{S}_{a} \cup \mathcal{S}_{b} \cup \mathcal{S}_{c} \cup \mathcal{S}_{d}: & \Delta_{1} \geq 0 \cap \Delta_{2}>0 \cap \Delta_{3}<0 & \text { if } \quad \kappa \in\left(\kappa_{3}^{\star}, \kappa_{4}^{\star}\right] \\
\mathcal{S}_{a} \cup \mathcal{S}_{b} \cup \mathcal{S}_{d}: & \Delta_{1}<0 \cap \Delta_{2} \geq 0 \cap \Delta_{3}<0 & \text { if } \quad \kappa \in\left(\kappa_{4}^{\star}, \kappa_{5}^{\star}\right] \\
\mathcal{S}_{a} \cup \mathcal{S}_{b}: & \Delta_{1}<0 \cap \Delta_{2}<0 \cap \Delta_{3}<0 & \text { if } \quad \kappa>\kappa_{5}^{\star}
\end{array}\right.
$$


where the conditions $\Delta_{i}(\kappa) \geq 0(i=1,2,3)$, with $\Delta_{i}$ given by Eqs. (23)-(25), are plotted in Fig. 2 , and the values of $\kappa_{j}^{\star}$ (with $j=1, \ldots, 5)$ are obtained by enforcing the condition $\Delta_{i}\left(\kappa_{j}^{\star}\right)=0$ in Eqs. (23)-(25). The result is

$$
\kappa_{1}^{\star}=1.466, \quad \kappa_{2}^{\star}=1.578, \quad \kappa_{3}^{\star}=1.607, \quad \kappa_{4}^{\star}=2.671, \quad \kappa_{5}^{\star}=3.606
$$

The obtained solution is confirmed by the contour lines of the required propulsive acceleration magnitude $\|\boldsymbol{u}\|$ shown in Fig. 3,

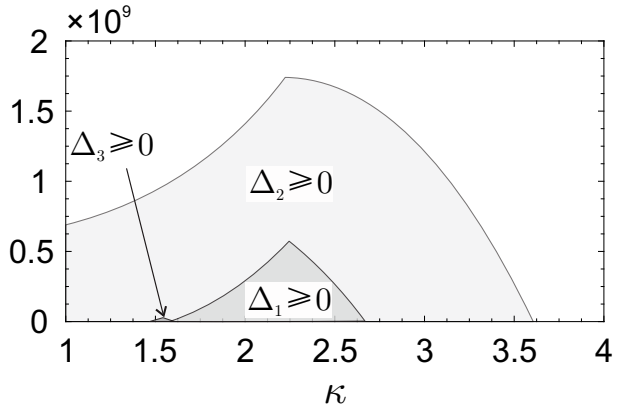

a) $\kappa \in[1,4]$

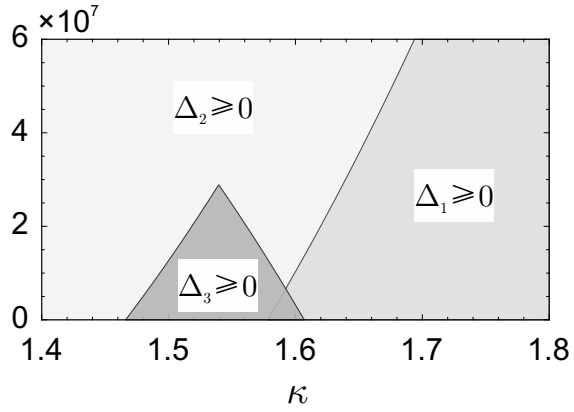

b) $\kappa \in[1.4,1.8]$

Figure 2 Regions of $\left\{\Delta_{1}, \Delta_{2}, \Delta_{3}\right\} \geq 0$ as a function of $\kappa$ : case of asteroid 433 Eros.

while the critical hovering directions $\left\{\theta^{\star}, \phi^{\star}\right\}$ are reassumed in Tab. 2.
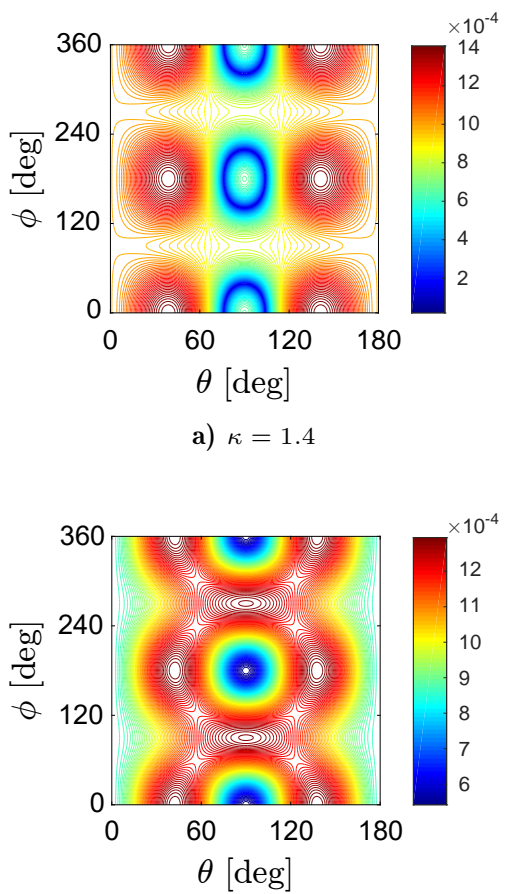

d) $\kappa=2$

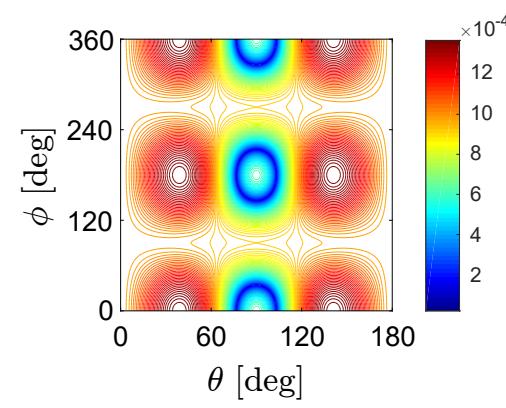

b) $\kappa=1.5$

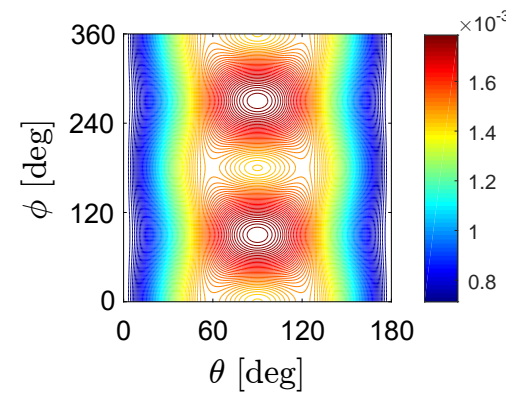

e) $\kappa=3$

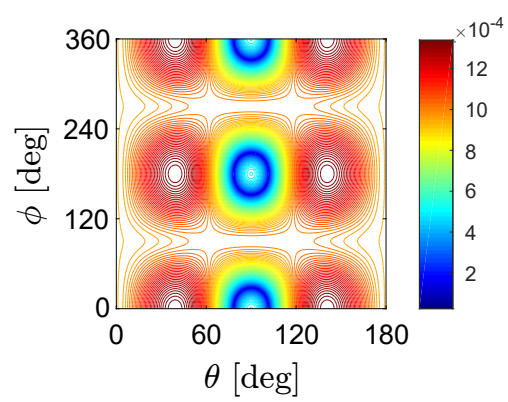

c) $\kappa=1.6$

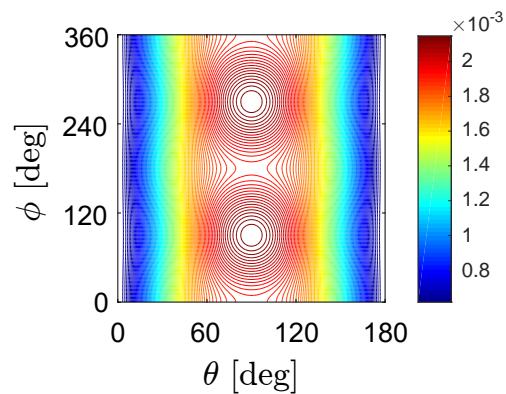

f) $\kappa=4$

Figure 3 Contour lines of required propulsive acceleration $\|\boldsymbol{u}\|\left[\mathrm{m} / \mathrm{s}^{2}\right]$ as a function of $\{\theta, \phi\}$ and $\kappa$ : case of asteroid 433 Eros.

Table 2 Critical hovering directions $\left\{\theta^{\star}, \phi^{\star}\right\}[\mathrm{deg}]$ of asteroid 433 Eros.

\begin{tabular}{|c|c|c|c|}
\hline$\kappa$ & $\mathcal{S}_{c}$ & $\mathcal{S}_{d}$ & $\mathcal{S}_{e}$ \\
\hline 1.4 & $\emptyset$ & $\begin{aligned} \theta^{\star} & =\{38.9,141.1\} \\
\phi^{\star} & =\{0,180,360\}\end{aligned}$ & $\emptyset$ \\
\hline 1.5 & $\emptyset$ & $\begin{aligned} \theta^{\star} & =\{39.0,141.0\} \\
\phi^{\star} & =\{0,180,360\}\end{aligned}$ & $\begin{aligned} \theta^{\star} & =\{28.4,151.6\}, \\
\phi^{\star} & =\{90,270\}\end{aligned}$ \\
\hline 1.6 & $\begin{aligned} \theta^{\star} & =\{61.7,118.3\} \\
\phi^{\star} & =\{84.0,96.0,264.0,276.0\}\end{aligned}$ & $\begin{aligned} \theta^{\star} & =\{39.3,140.7\} \\
\phi^{\star} & =\{0,180,360\}\end{aligned}$ & $\begin{aligned} \theta^{\star} & =\{76.7,103.3\}, \\
\phi^{\star} & =\{90,270\}\end{aligned}$ \\
\hline 2 & $\begin{aligned} \theta^{\star} & =\{56.8,123.2\} \\
\phi^{\star} & =\{58.4,121.6,238.4,301.6\}\end{aligned}$ & $\begin{aligned} \theta^{\star} & =\{42.3,137.7\} \\
\phi^{\star} & =\{0,180,360\}\end{aligned}$ & $\emptyset$ \\
\hline 3 & $\emptyset$ & $\begin{aligned} \theta^{\star} & =\{59.4,120.6\} \\
\phi^{\star} & =\{0,180,360\}\end{aligned}$ & $\emptyset$ \\
\hline 4 & $\emptyset$ & $\emptyset$ & $\emptyset$ \\
\hline
\end{tabular}




\section{Minimum and Maximum Fuel-Consumption Directions}

Despite the critical hovering directions corresponding to fuel-consumption extrema may be found with an analytical approach, the important question remains on how the hovering directions must be chosen within the admissible set to guarantee a globally optimal solution. In fact, since some local fuel-consumption extrema may exist (see Fig. 3), the conditions for obtaining a global minimum or a maximum require a further study.

To that end, Eqs. (18)-(22) should be considered together with Fig. 3 for a more direct insight into the variation tendency of the global extrema. Note that, as long as $\kappa$ is less than a critical value $\kappa_{a}^{\star}$, the minimum fuel-consumption hovering always happens on the $\hat{\boldsymbol{x}}$-axis, that is, $\theta_{a 1}^{\star}=\pi / 2$ and $\phi_{a 1}^{\star}=\{0, \pi, 2 \pi\}$. On the contrary, when $\kappa>\kappa_{a}^{\star}$, the critical hovering directions become $\theta_{a 2}^{\star}=\{0, \pi\}$, with $\phi_{a 2}^{\star}$ arbitrary, showing that the fuel consumption takes its global minimum on the $\hat{z}$-axis. In summary, the minimum required acceleration can be written as

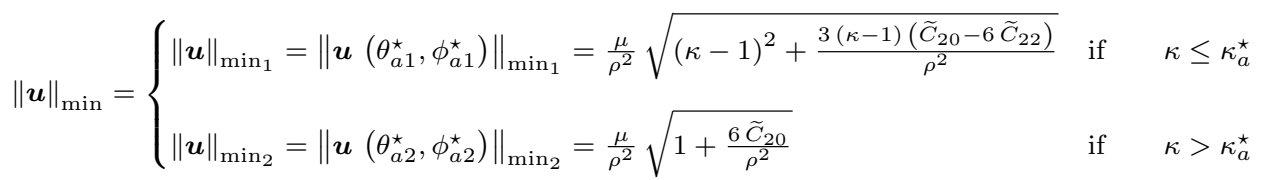

The value of $\kappa_{a}^{\star}$ may be found by enforcing the condition $\|\boldsymbol{u}\|_{\min _{1}}=\|\boldsymbol{u}\|_{\min _{2}}$, thus obtaining an algebraic equation in one unknown, that is

$$
\kappa_{a}^{\star}\left(\kappa_{a}^{\star}-2\right)\left(\frac{\mu \kappa_{a}^{\star}}{\omega^{2}}\right)^{2 / 3}+3\left(\widetilde{C}_{20}-6 \widetilde{C}_{22}\right)\left(\kappa_{a}^{\star}-1\right)-6 \widetilde{C}_{20}=0
$$

which is easily solved numerically for $\kappa_{a}^{\star}$. For example, using the data of asteroid 433 Eros, it turns out that $\kappa_{a}^{\star}=2.2267$.

The maximum fuel-consumption hovering case can be studied in a similar way. As long as $\kappa \leq \kappa_{b}^{\star}$, the maxima are reached when the critical directions lie on the $(\hat{\boldsymbol{x}}, \hat{\boldsymbol{z}})$ plane. In that case, $\left\{\theta_{b 1}^{\star}, \phi_{b 1}^{\star}\right\} \in \mathcal{S}_{d}$, see Eq. (21). On the other hand, if $\kappa>\kappa_{b}^{\star}$, the maximum fuel-consumption hovering condition always takes place on the $\hat{\boldsymbol{y}}$-axis, i.e. when $\theta_{b 2}^{\star}=\pi / 2$ and $\phi_{b 2}^{\star}=\{\pi / 2,3 \pi / 2\}$, viz.

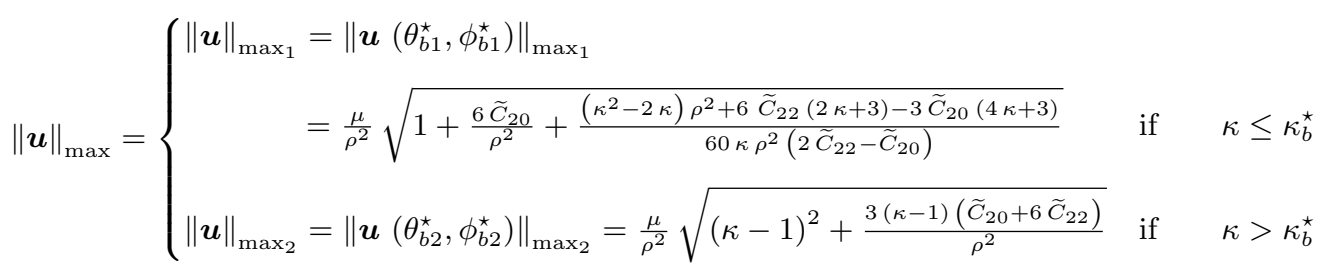

The value of $\kappa_{b}^{\star}$ satisfies the equality $\|\boldsymbol{u}\|_{\max _{1}}=\|\boldsymbol{u}\|_{\max _{2}}$ and, for asteroid 433 Eros, the value is $\kappa_{b}^{\star}=2.0203$.

The values of $\left\{\|\boldsymbol{u}\|_{\min },\|\boldsymbol{u}\|_{\max }\right\}$ obtained from Eqs. (28) and (30) are validated by Fig. 4, which shows the projection of the contour lines of $\|\boldsymbol{u}\|$ when $\kappa<\kappa_{b}^{\star}, \kappa=\kappa_{b}^{\star}, \kappa=\kappa_{a}^{\star}$, and $\kappa>\kappa_{a}^{\star}$. Note that the numerical values of minima (denoted with a circle) and maxima (denoted with a star) are consistent with the analytical results, thus reinforcing the soundness of the presented method.

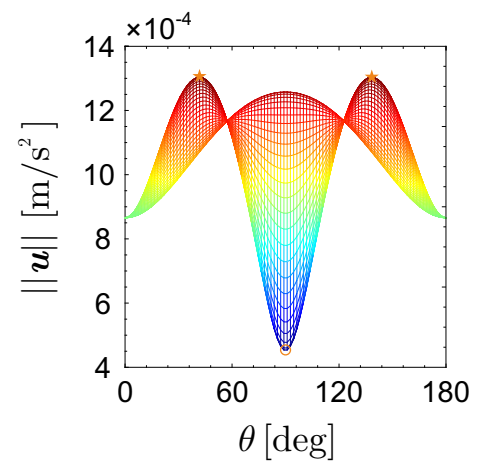

a) $\kappa=1.95<\kappa_{b}^{\star}$

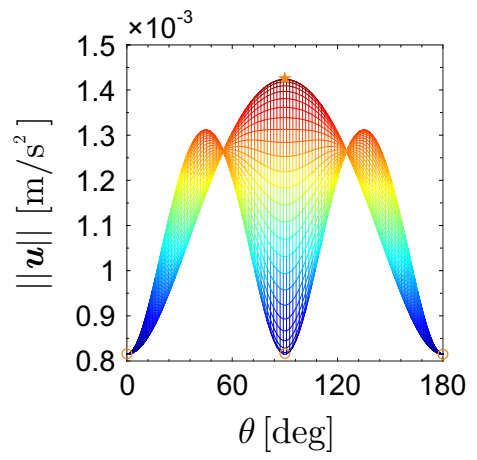

c) $\kappa=\kappa_{a}^{\star}$

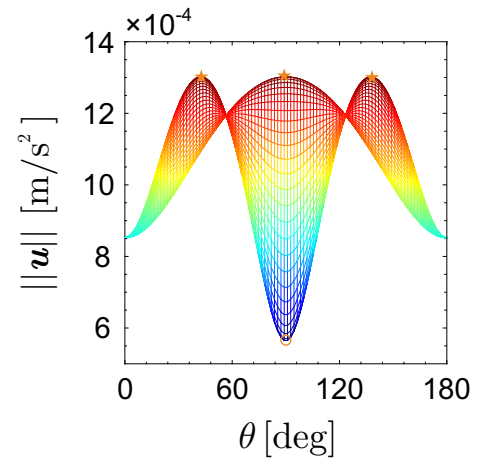

b) $\kappa=\kappa_{b}^{\star}$

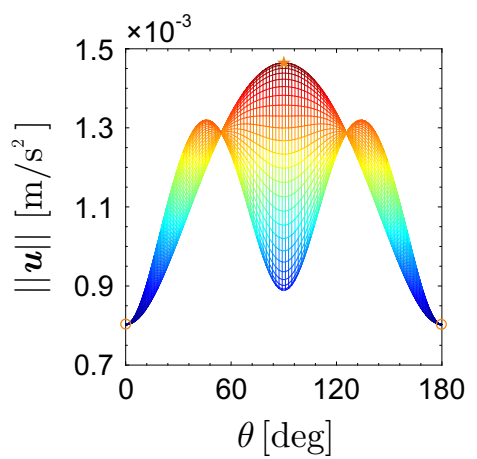

d) $\kappa=2.3>\kappa_{a}^{\star}$

Figure 4 Contour lines of magnitude $\|\boldsymbol{u}\|$ projected to the $(\hat{\boldsymbol{x}}, \hat{\boldsymbol{z}})$ plane when $\kappa<\kappa_{b}^{\star}, \kappa=\kappa_{b}^{\star}, \kappa=\kappa_{a}^{\star}$, and $\kappa>\kappa_{a}^{\star}$. 


\section{Stability Analysis}

The previous analysis provides a basis on which potential asteroid hovering positions can be chosen from a fuel-consumption viewpoint. However, the practical implementation of a hovering mission would be greatly simplified by the choice of a stable hovering point. For this reason, the stability of asteroid hovering problem, with a qualitative description in terms of parameter $\kappa$, will now be investigated. The problem is addressed by assuming that the actual spacecraft position vector differs from the designed hovering position by a small quantity. Let $\delta \boldsymbol{r} \triangleq[\delta x, \delta y, \delta z]^{\mathrm{T}}$ be the deviation in hovering position, and $V \triangleq-(\boldsymbol{\omega} \times \boldsymbol{r})(\boldsymbol{\omega} \times \boldsymbol{r}) / 2+U$ the effective potential function. Because $\|\delta \boldsymbol{r}\|$ may be considered small when compared to the asteroid-spacecraft distance $\|\boldsymbol{r}\|$, the linearized equation describing the error dynamics around the hovering position is obtained from Eq. (1) as

$$
\delta \ddot{\boldsymbol{r}}+2 \boldsymbol{\omega} \times \delta \dot{\boldsymbol{r}}+\frac{\partial^{2} V}{\partial \boldsymbol{r}^{2}}=\mathbf{0}
$$

in which the variation in propulsive acceleration $\delta \boldsymbol{u} \simeq 0$ is neglected. Equation (31) gives

$$
\begin{array}{r}
\delta \ddot{x}-2 \omega \delta \dot{y}+V_{x x} \delta x+V_{x y} \delta y+V_{x z} \delta z=0 \\
\delta \ddot{y}+2 \omega \delta \dot{x}+V_{y x} \delta x+V_{y y} \delta y+V_{y z} \delta z=0 \\
\delta \ddot{z}+V_{z x} \delta x+V_{z y} \delta y+V_{z z} \delta z=0
\end{array}
$$

where $V_{i j}=V_{j i}=\partial^{2} V / \partial i \partial j$, with $i, j=\{x, y, z\}$, is the generic entry of $\partial^{2} V / \partial \boldsymbol{r}^{2}$, whose expression is omitted here for brevity.

The motion stability around the hovering position may be investigated, as usual, by applying the Laplace transform to the set of linear differential equations (32)-(34). It may be verified that the resulting characteristic polynomial is

$$
s^{6}+\mathcal{C}_{2} s^{4}+\mathcal{C}_{1} s^{2}+\mathcal{C}_{0}=0
$$

where $s$ is the Laplace variable, and the coefficients of the characteristic polynomial are

$$
\begin{aligned}
& \mathcal{C}_{0}=V_{x x} V_{y y} V_{z z}+2 V_{x y} V_{x z} V_{y z}-V_{x x} V_{y z}^{2}-V_{y y} V_{x z}^{2}-V_{z z} V_{x y}^{2} \\
& \mathcal{C}_{1}=V_{x x} V_{y y}+V_{x x} V_{z z}+V_{y y} V_{z z}-V_{x y}^{2}-V_{x z}^{2}-V_{y z}^{2}+4 \omega^{2} V_{z z} \\
& \mathcal{C}_{2}=V_{x x}+V_{y y}+V_{z z}+4 \omega^{2}
\end{aligned}
$$

The stability of the linear system is determined by the roots of the characteristic equation (35), which can be rewritten as

$$
\eta^{3}+\mathcal{C}_{2} \eta^{2}+\mathcal{C}_{1} \eta+\mathcal{C}_{0}=0
$$

where $\eta \triangleq s^{2}$. Because Eq. (39) is a cubic polynomial of $\eta$, its roots are either all real or one root is real and the other two are complex. According to the topological property of the solution [8], the hovering position is stable if and only if all of the roots of Eq. (39) are non-positive real numbers. In fact, in correspondence of a complex root of Eq. (39), there would be a (unstable) root with positive real part in Eq. (35). Therefore, the necessary and sufficient conditions for linear stability are

$$
\Delta_{s} \leq 0 \cap \mathcal{C}_{0} \geq 0 \quad \cap \quad \mathcal{C}_{1} \geq 0 \quad \cap \quad \mathcal{C}_{2} \geq 0
$$

where

$$
\Delta_{s}=\left(\mathcal{C}_{1} \mathcal{C}_{2}-9 \mathcal{C}_{0}\right)^{2}-4\left(\mathcal{C}_{2}^{2}-3 \mathcal{C}_{1}\right)\left(\mathcal{C}_{1}^{2}-3 \mathcal{C}_{0} \mathcal{C}_{2}\right)
$$

In Eq. (40), the condition $\Delta_{s} \leq 0$ implies the existence of three real roots of Eq. (39), while the last three conditions are enforced to guarantee that all the roots of Eq. (39) are non-positive.

For illustrative purposes, Fig. 5 shows the (linearly) stable hovering regions over asteroid 433 Eros; see also Tab. 1 . A value of $\kappa=2$ has been chosen, which corresponds to a hovering distance of about $\rho=20.12 \mathrm{~km}$. To qualitatively visualize the effect of parameter $\kappa$, the stable regions with a continuation of $\kappa$ are illustrated in Fig. 6 when $\kappa=\{1.75,1.8,2.5,3,4,5\}$. As expected, the stability regions expand as $\kappa$ increases, even though further simulations show that such an increase becomes small when $\kappa$ is greater than about 4.13 .

\section{Fuel-Optimal Transfer Toward a Given Hovering Point}

\section{Homotopic Method for Trajectory Optimization}

In this section the transfer problem toward a hovering point is formulated in an optimal framework, that is, by minimizing the fuel required to complete the transfer. To that end, consider a spacecraft of mass $m$, whose primary propulsion system is an electric thruster with constant specific impulse $I_{\mathrm{sp}}$, and maximum thrust magnitude $T_{\max }$. Taking into account the asteroid gravity and the propulsion system thrust only, and bearing in mind Eq. (1), the spacecraft equations of motion in the rotating frame $\mathcal{T}$ are

$$
\begin{aligned}
\dot{\boldsymbol{r}} & =\boldsymbol{v} \\
\dot{\boldsymbol{v}} & =-2 \boldsymbol{\omega} \times \boldsymbol{v}-\frac{\partial V}{\partial \boldsymbol{r}}+\frac{T_{\max } \tau}{m} \hat{\boldsymbol{\alpha}} \\
\dot{m} & =-\frac{T_{\max } \tau}{I_{\mathrm{sp}} g_{0}}
\end{aligned}
$$




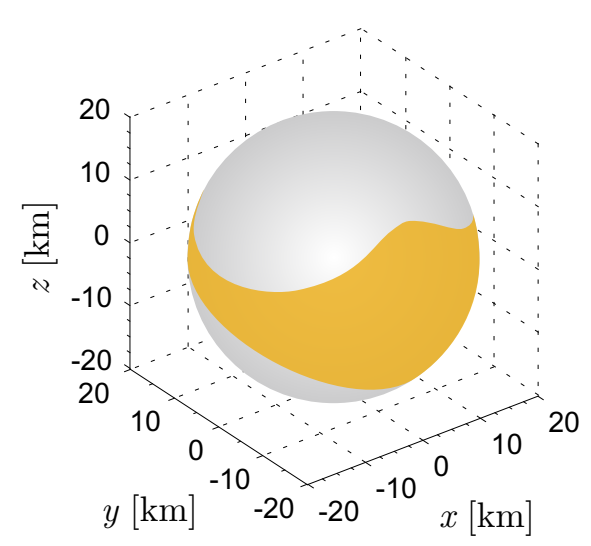

a) $3 \mathrm{D}$ view.

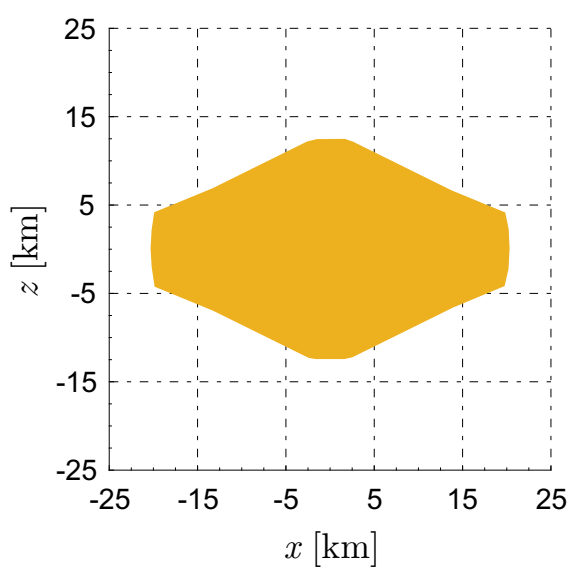

c) $(\hat{\boldsymbol{x}}-\hat{\boldsymbol{z}})$ plane projection.

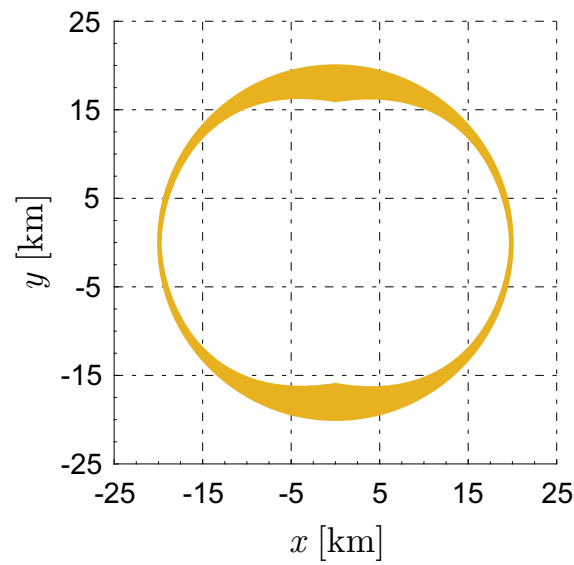

b) $(\hat{\boldsymbol{x}}-\hat{\boldsymbol{y}})$ plane projection

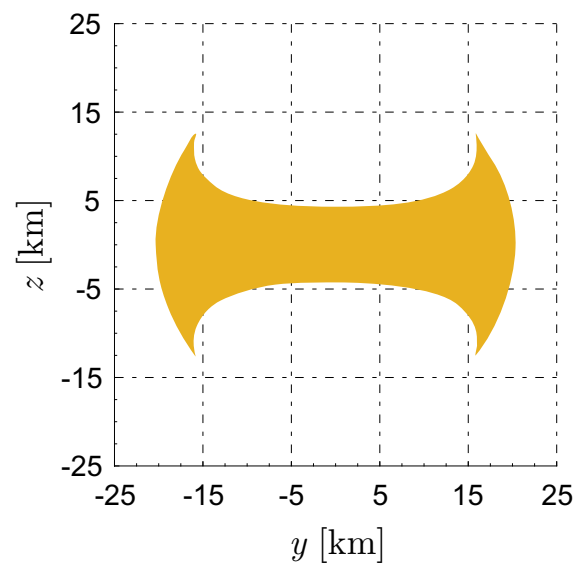

d) $(\hat{\boldsymbol{y}}-\hat{\boldsymbol{z}})$ plane projection.

Figure 5 Linear stable regions when $\kappa=2$ : case of asteroid 433 Eros.

where $g_{0}$ is the standard gravity, $\tau \in[0,1]$ is the engine throttle level, and $\hat{\boldsymbol{\alpha}}$ is the unit thrust vector. The problem addressed here consists in finding the optimal control law $\boldsymbol{a}^{\star}=\boldsymbol{a}\left(\tau^{\star}, \hat{\boldsymbol{\alpha}}^{\star}\right)$ that minimizes the fuel required to transfer the spacecraft from an arbitrary initial state $\left(\boldsymbol{r}_{0}, \boldsymbol{v}_{0}\right)$ to a final desired hovering point $\left(\boldsymbol{r}_{f}, \boldsymbol{v}_{f}=\mathbf{0}\right)$, within a given flight time $t_{f}$. The performance index to minimize is

$$
J_{0}=\frac{T_{\max }}{I_{\mathrm{sp}} g_{0}} \int_{0}^{t_{f}} \tau \mathrm{d} t
$$

in which $t_{f}$ is fixed.

The optimal control problem is solved through an indirect approach. The fuel-optimal problem, which suffers from a rather limited convergence range and a high sensitivity to the initial guess, is first translated into an energy-optimal problem, which is easier to solve due to its inherent continuity and differentiability. Thus, instead of the original optimization index given by Eq. (45), the homotopy here is built by introducing the modified perturbed performance index [20]

$$
J=\frac{\lambda_{0} T_{\max }}{I_{\mathrm{sp}} g_{0}} \int_{0}^{t_{f}}[\tau-\epsilon \tau(1-\tau)] \mathrm{d} t
$$

where $\epsilon \in[0,1]$ is the homotopy parameter that links the fuel-optimal criteria $(\epsilon=0)$ with the energy-optimal criteria $(\epsilon=1)$, and $\lambda_{0} \in \mathbb{R}^{+}$is a scaling factor used for restricting the costate vector on a unit hypersphere. From a practical standpoint, the energy-optimal problem is first solved with a shooting method, and then $\epsilon$ is decreased with a continuation procedure until the solution of the fuel-optimal problem is eventually obtained.

Bearing in mind Eqs. (42)-(44), the Hamiltonian $\mathcal{H}$ can be written as

$$
\mathcal{H}=\boldsymbol{\lambda}_{r} \cdot \boldsymbol{v}+\boldsymbol{\lambda}_{v} \cdot\left(-2 \boldsymbol{\omega} \times \boldsymbol{v}-\frac{\partial V}{\partial \boldsymbol{r}}+\frac{T_{\max } \tau}{m} \hat{\boldsymbol{\alpha}}\right)-\frac{\lambda_{m} T_{\max } \tau}{I_{\mathrm{sp}} g_{0}}+\frac{\lambda_{0} T_{\max }[\tau-\epsilon \tau(1-\tau)]}{I_{\mathrm{sp}} g_{0}}
$$

where $\boldsymbol{\lambda}_{r}$ and $\boldsymbol{\lambda}_{v}$ are the costate vectors adjoint to the position and the velocity, respectively, and $\lambda_{m}$ is the mass costate. 


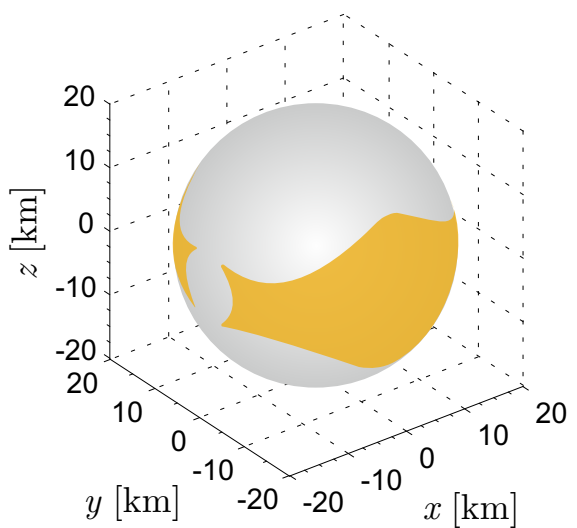

a) $\kappa=1.75$

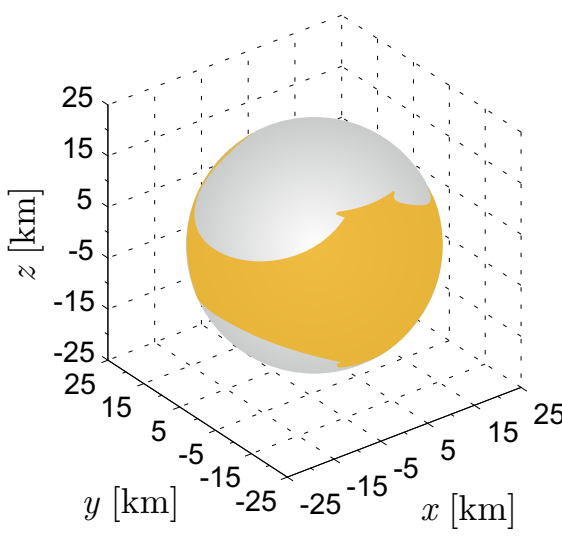

c) $\kappa=2.5$

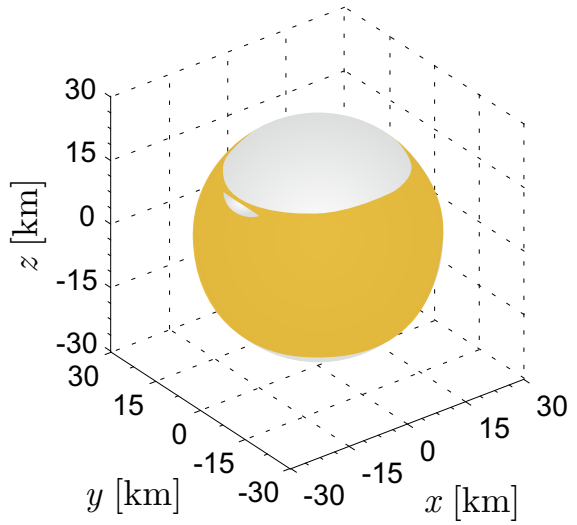

e) $\kappa=4$

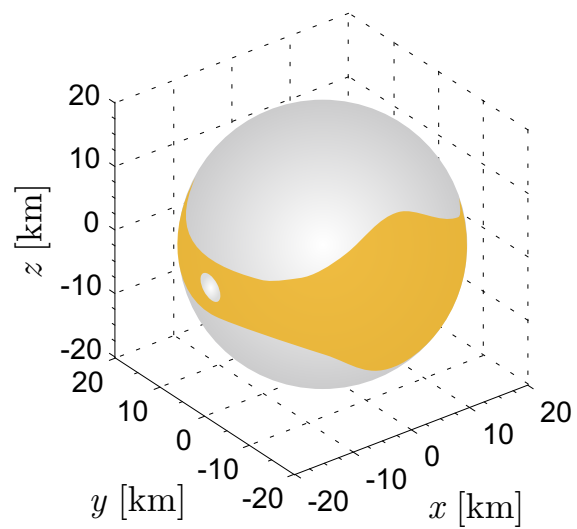

b) $\kappa=1.8$

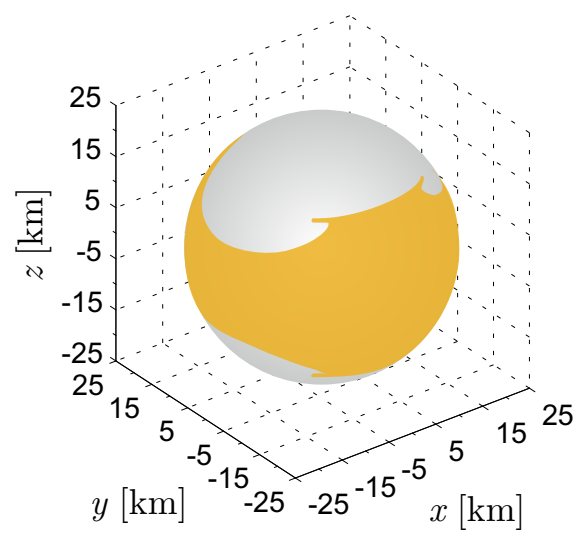

d) $\kappa=3$

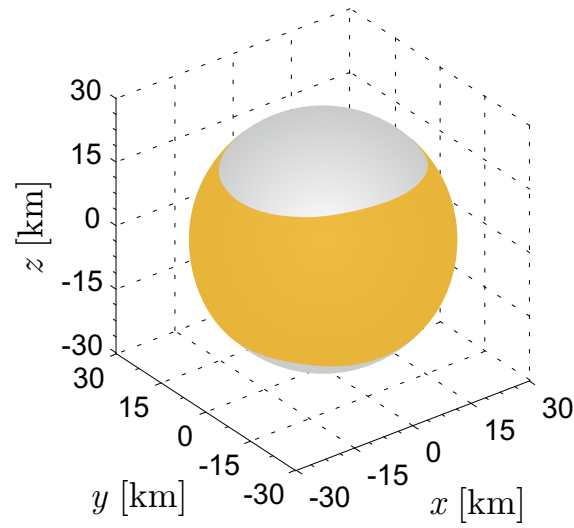

f) $\kappa=5$

Figure 6 Linear stable regions as a function of $\kappa$ : case of asteroid 433 Eros.

Accordingly, the Euler-Lagrange equations are

$$
\begin{aligned}
& \dot{\boldsymbol{\lambda}}_{r}=-\frac{\partial \mathcal{H}}{\partial \boldsymbol{r}}=\boldsymbol{\omega} \times\left(\boldsymbol{\omega} \times \boldsymbol{\lambda}_{v}\right)+\frac{\partial^{2} U}{\partial \boldsymbol{r}^{2}} \cdot \boldsymbol{\lambda}_{v} \\
& \dot{\boldsymbol{\lambda}}_{v}=-\frac{\partial \mathcal{H}}{\partial \boldsymbol{v}}=-\boldsymbol{\lambda}_{r}+2 \boldsymbol{\lambda}_{v} \times \boldsymbol{\omega} \\
& \dot{\lambda}_{m}=-\frac{\partial \mathcal{H}}{\partial m}=\frac{T_{\max } \tau}{m^{2}} \boldsymbol{\lambda}_{v} \cdot \hat{\boldsymbol{\alpha}}
\end{aligned}
$$

According to the Pontryagin's maximum principle, the optimal control law $\boldsymbol{a}^{\star}=\boldsymbol{a}\left(\tau^{\star}, \hat{\boldsymbol{\alpha}}^{\star}\right)$, to be selected in the feasible control domain $\mathcal{D}$, is designed such that the Hamiltonian is an absolute minimum at any time, that is

$$
\boldsymbol{a}^{\star}=\arg \min _{\boldsymbol{a} \in \mathcal{D}} \mathcal{H}
$$


Therefore, the optimal thrust vector $\hat{\boldsymbol{\alpha}}^{\star}$ that minimizes the Hamiltonian $\mathcal{H}$ is

$$
\hat{\boldsymbol{\alpha}}^{\star}=-\frac{\boldsymbol{\lambda}_{v}}{\left\|\boldsymbol{\lambda}_{v}\right\|}
$$

and the optimal throttle level $\tau^{\star}$ is

$$
\tau^{\star}=\left\{\begin{array}{lll}
0 & \text { if } & \beta>\epsilon \\
1 & \text { if } & \beta<-\epsilon \\
\frac{\epsilon-\beta}{2 \epsilon} & \text { if } & |\beta| \leq \epsilon
\end{array}\right.
$$

where $\beta$ is the switching function, defined as

$$
\beta=1-\frac{I_{\mathrm{sp}} g_{0}\left\|\boldsymbol{\lambda}_{v}\right\|}{\lambda_{0} m}-\frac{\lambda_{m}}{\lambda_{0}}
$$

Since no constraint is enforced on the final mass, the transversality condition gives $\lambda_{m}\left(t_{f}\right)=0$. To further alleviate the difficulties in solving the TPBVP, a normalization technique of the initial costate vector is employed. To this end, introduce a new costate vector

$$
\boldsymbol{\lambda} \triangleq\left[\boldsymbol{\lambda}_{r}^{\mathrm{T}}, \boldsymbol{\lambda}_{v}^{\mathrm{T}}, \lambda_{m}, \lambda_{0}\right]^{\mathrm{T}}
$$

Note that the Hamiltonian $\mathcal{H}$ is a homogeneous function of $\boldsymbol{\lambda}$, so that the components of the costate variables $\boldsymbol{\lambda}$ can be normalized to range within the interval $[-1,1]$, viz.

$$
\|\boldsymbol{\lambda}(0)\|=\sqrt{\left\|\boldsymbol{\lambda}_{r}(0)\right\|^{2}+\left\|\boldsymbol{\lambda}_{v}(0)\right\|^{2}+\lambda_{m}(0)^{2}+\lambda_{0}^{2}}=1
$$

Finally, with the new boundary condition (56), the shooting function of the TPBVP in terms of $\boldsymbol{\lambda}(0)$ is

$$
\boldsymbol{\Phi}[\boldsymbol{\lambda}(0)]=\left[\boldsymbol{r}^{\mathrm{T}}\left(t_{f}\right)-\boldsymbol{r}_{f}^{\mathrm{T}}, \boldsymbol{v}^{\mathrm{T}}\left(t_{f}\right), \lambda_{m}\left(t_{f}\right),\|\boldsymbol{\lambda}(0)\|-1\right]^{\mathrm{T}}
$$

A continuation procedure parameterized with $\epsilon$, which decreases from 1 to 0 , is adopted to target the initial costate vector $\boldsymbol{\lambda}(0)$ for the fuel-optimal problem $(\epsilon=0)$.

\section{Case Study}

Consider a spacecraft with an initial mass $m_{0}=2000 \mathrm{~kg}$, a maximum thrust magnitude $T_{\max }=60 \mathrm{~N}$, and a specific impulse $I_{\mathrm{sp}}=400 \mathrm{~s}$. The acceleration magnitude of the electric thruster here is in accordance with the maximum value of that in an asteroid descent mission [21]. The optimal control law with a homotopy mapping-based algorithm is used to study the fuel-optimal trajectories for the case of asteroid 433 Eros; see Tab. 1. In particular, a typical mission scenario consisting in transferring the spacecraft to a preferable hovering point is addressed. The spacecraft initial position is $\boldsymbol{r}_{0}=[30.3,17.5,0]^{\mathrm{T}} \mathrm{km}$, the final position is $\boldsymbol{r}_{f}=[20.12,0,0]^{\mathrm{T}} \mathrm{km}$, while the initial and final velocities are assumed to be both zero. Note that for a hovering distance $\rho=20.12 \mathrm{~km}$, the parameter $\kappa$ is equal to 2 , which gives a linearly stable and minimum fuel-consumption hovering position over asteroid 433 Eros, as shown in Fig. 3 and Fig. 5.

A set of fuel-optimal transfer trajectories are collected in Fig. 7 as a function of the flight time $t_{f} \in[1680,4000] \mathrm{s}$, while the value of the spacecraft final mass $m_{f}=m\left(t_{f}\right)$ is shown in Fig. 8. Note that the final mass $m_{f}$ is, as expected, positively correlated with the given flight time $t_{f}$ according to Eq. (44).

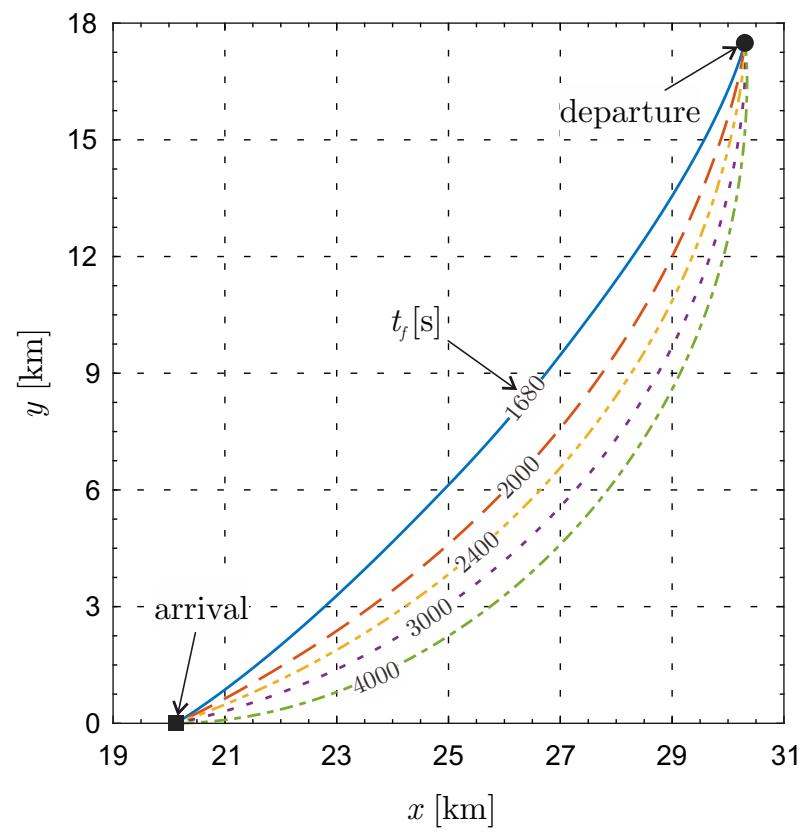

Figure 7 Fuel-optimal transfer trajectories as a function of $t_{f}$.

The simulations imply that the value of the minimum flight time that allows for a feasible solution is about $t_{f \min } \simeq 1672 \mathrm{~s}$. For example, assuming a flight time $t_{f}=2000 \mathrm{~s}$, the time variation of the spacecraft mass is given in Fig. 9, which shows the presence of a coasting arc (i.e., a phase in which $\tau^{\star}=0$ ) of about $1070 \mathrm{~s}$ in the optimal trajectory. 


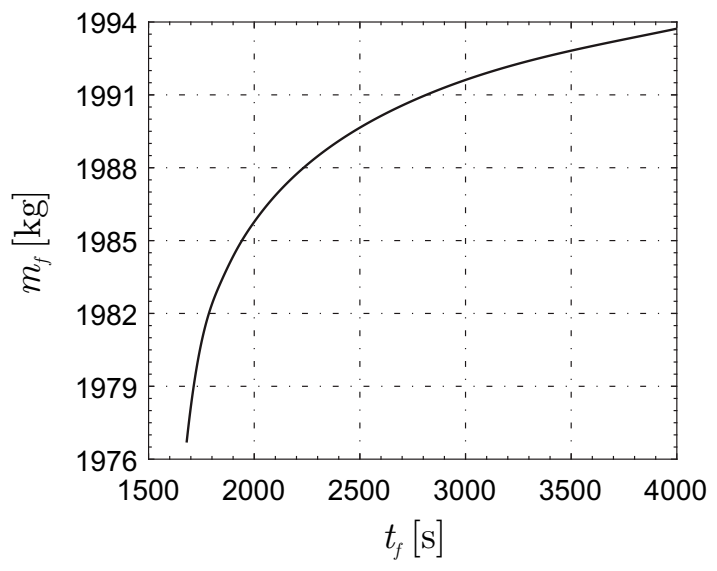

Figure 8 Final spacecraft mass $m_{f}$ as a function of $t_{f}$.

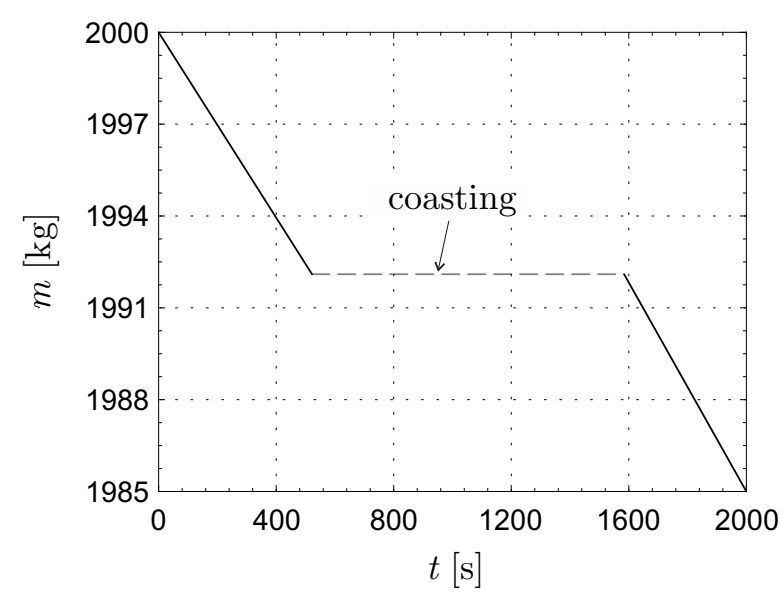

Figure 9 Time variation of the spacecraft $m$ when $t_{f}=2000 \mathrm{~s}$.

The throttle level $\tau$ during a homotopic process (where $\epsilon$ is discretized with a step of 0.2) is illustrated in Fig. 10, showing that a bang-bang control law is eventually obtained when $\epsilon=0$. Finally, Fig. 11 provides the time variation of the thrust

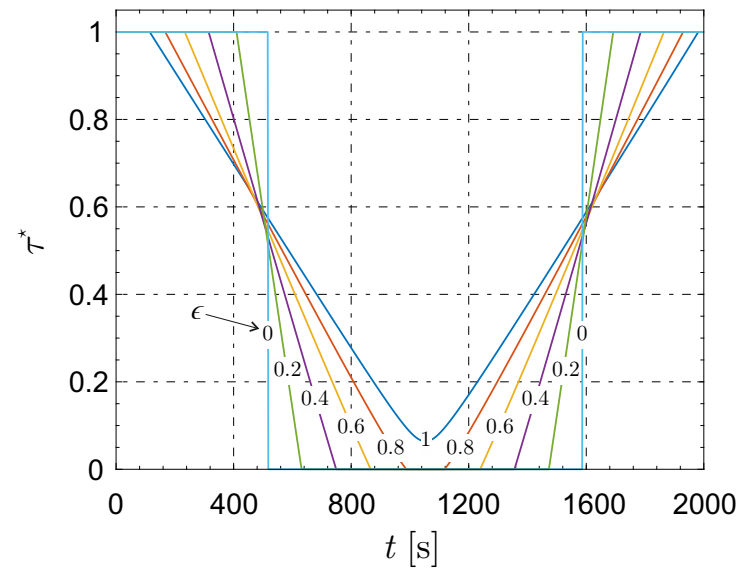

Figure 10 Optimal throttle level $\tau^{\star}$ as a function of $\epsilon$ when $t_{f}=2000 \mathrm{~s}$.

vector components. Note that the out-of-plane component is $T_{z}=0$ (recall that both the initial and final position of the spacecraft lies in the $(\hat{\boldsymbol{x}}, \hat{\boldsymbol{y}})$ plane), which states that the optimal transfer trajectory is planar.

\section{Conclusions}

The problem of asteroid hovering by a spacecraft equipped with a continuous-thrust propulsion system has been thoroughly investigated from a fuel-consumption perspective. Under the assumption of a second-degree and a second-order non-spherical gravitational field, it has been shown that many fuel-consumption extrema may exist for asteroid hovering missions. The closed-form solutions of the critical directions and the corresponding fuel-consumption extrema have been found through an approximate method, while the global minima and maxima have been calculated with an analytical approach.

The linear stability for a given hovering distance has been studied in a parametric way, showing that the stable region enlarges as the hovering distance increases. In particular, the optimal steering law that minimizes the fuel consumption toward a stable hovering point has been analyzed using an indirect approach, in which a homotopic method (with an initial costate vector normalization technique) has been adopted to reduce the numerical sensitivity of the optimal control problem. For 


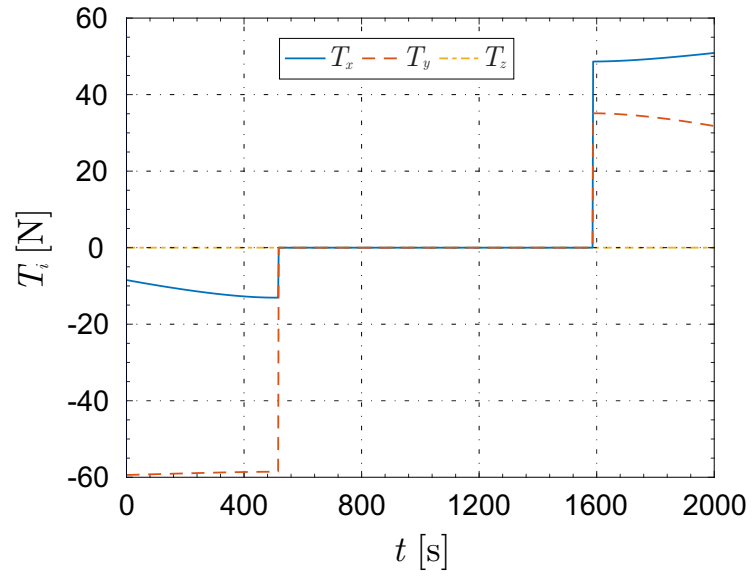

Figure 11 Time variation of the thrust vector components in $\mathcal{T}$ when $t_{f}=2000 \mathrm{~s}$.

the purpose of decreasing the propellant expense, the minimum fuel-consumption hovering directions are preferable, provided that the mission requirements allow some flexibility in specifying the hovering site. The fuel-optimal transfer can be used as a nominal reference trajectory of a spacecraft in practical operations.

\section{Acknowledgment}

This work was supported by the National Natural Science Foundation of China (Grant nos. 11525208 and 11902175), Postdoctoral Science Foundation of China (Grant nos. BX20190166 and 2019M650700), and University of Pisa, Progetti di Ricerca di Ateneo (Grant no. PRA_2018_44).

\section{References}

[1] Scheeres, D. J., Orbital Motion in Strongly Perturbed Environments: Applications to Asteroid, Comet and Planetary Satellite Orbiters, chap. 1-2, Springer-Praxis, London, UK, 2012, pp. 3-22, 41-52, ISBN: 978-3-642-03255-4.

[2] Wie, B., Zimmerman, B., Lyzhoft, J., and Vardaxis, G., "Planetary Defense Mission Concepts for Disrupting/Pulverizing Hazardous Asteroids with Short Warning Time," Astrodynamics, Vol. 1, No. 1, September 2016, pp. 3-21. doi: 10.1007/s42064-017-0002-9.

[3] Acuña, M. H., Russell, C. T., Zanetti, L. J., and Anderson, B. J., "The NEAR Magnetic Field Investigation: Science Objectives at Asteroid Eros 433 and Experimental Approach," Journal of Geophysical Research: Planets, Vol. 102, No. E10, October 1997, pp. 23751-23759. doi: doi.org/10.1029/97JE01161.

[4] Baker, J., "The Falcon Has Landed," Science, Vol. 312, No. 5778, June 2006, pp. 1327. doi: 10.1126/science.312.5778.1327.

[5] Xu, M., Liang, Y., and Ren, K., "Survey on Advances in Orbital Dynamics and Control for Libration Point Orbits," Progress in Aerospace Sciences, Vol. 82, April 2016, pp. 24-35. doi: 10.1016/j.paerosci.2015.12.005.

[6] Wang, W., Mengali, G., Quarta, A. A., and Yuan, J., "Analysis of Relative Motion in Non-Keplerian Orbits via Modified Equinoctial Elements," Aerospace Science and Technology, Vol. 58, No. 8, November 2016, pp. 389-400. doi: 10.1016/j.ast.2016.09.001.

[7] Wang, W., Quarta, A. A., Mengali, G., and Yuan, J., "Multiple Solar Sail Formation Flying Around Heliocentric Displaced Orbit via Consensus," Acta Astronautica, Vol. 154, November 2019, pp. 256-267. doi: 10.1016/j.actaastro.2018.03.039.

[8] Yang, H., Bai, X., and Li, S., "Artificial Equilibrium Points near Irregular-Shaped Asteroids with Continuous Thrust," Journal of Guidance, Control, and Dynamics, Vol. 41, No. 6, November-December 2018, pp. 1308-1319. doi: 10.2514/1.G003295.

[9] Gong, S. and Li, J., "Equilibria Near Asteroids for Solar Sails with Reflection Control Devices," Astrophysics and Space Science, Vol. 355, No. 2, February 2015, pp. 213-223. doi: 10.1007/s10509-014-2165-7.

[10] Zeng, X., Gong, S., Li, J., and Alfriend, K. T., "Solar Sail Body-Fixed Hovering over Elongated Asteroids," Journal of Guidance, Control, and Dynamics, Vol. 39, No. 6, June 2016, pp. 1223-1231. doi: 10.2514/1.G001061.

[11] Zhang, Y., Zeng, X., and Zhang, F., "Spacecraft Hovering Flight in a Binary Asteroid System by Using Fuzzy Logic Control," IEEE Transactions on Aerospace and Electronic Systems, Vol. 55, No. 6, December 2019, pp. 3246-3258. doi: 10.1109/TAES.2019.2906435.

[12] Guelman, M., "Closed-Loop Control of Close Orbits Around Asteroids," Journal of Guidance, Control, and Dynamics, Vol. 38, No. 5, May 2015, pp. 854-860. doi: 10.2514/1.G000158.

[13] Zhang, B. and Cai, Y., "Immersion and Invariance Based Adaptive Backstepping Control for Body-Fixed Hovering Over an Asteroid," IEEE Access, Vol. 7, March 2019, pp. 34850-34861. doi: 10.1109/ACCESS.2019.2904590.

[14] Gui, H. and Ruiter, A. H. J., "Control of Asteroid-Hovering Spacecraft with Disturbance Rejection Using Position-Only Measurements," Journal of Guidance, Control, and Dynamics, Vol. 40, No. 10, October 2017, pp. 2401-2416. doi: 10.2514/1.G002617.

[15] Furfaro, R., "Hovering in Asteroid Dynamical Environments Using Higher-Order Sliding Control," Journal of Guidance, Control, and Dynamics, Vol. 38, No. 2, February 2015, pp. 263-279. doi: 10.2514/1.G000631.

[16] Broschart, S. B. and Scheeres, D. J., "Boundedness of Spacecraft Hovering Under Dead-Band Control in Time-Invariant Systems," Journal of Guidance, Control, and Dynamics, Vol. 30, No. 2, March-April 2007, pp. 601-610. doi: 10.2514/1.20179.

[17] Sawai, S., Scheeres, D. J., and Broschart, S. B., "Control of Hovering Spacecraft Using Altimetry," Journal of Guidance, Control, and Dynamics, Vol. 25, No. 4, July-August 2002, pp. 786-795. doi: 10.2514/2.4947.

[18] Broschart, S. B. and Scheeres, D. J., "Control of Hovering Spacecraft Near Small Bodies: Application to Asteroid 25143 Itokawa," Journal of Guidance, Control, and Dynamics, Vol. 28, No. 2, November-December 2005, pp. 343-354. doi: 10.2514/1.3890.

[19] Bryson, A. E. and Ho, Y. C., Applied Optimal Control, chap. 1, Hemisphere Publishing Corporation, New York, NY, 1975, pp. 9-18, ISBN: 0-891-16228-3.

[20] Jiang, F., Baoyin, H., and Li, J., "Practical Techniques for Low-Thrust Trajectory Optimization with Homotopic Approach," Journal of Guidance, Control, and Dynamics, Vol. 35, No. 1, January-February 2012, pp. 245-257. doi: 10.2514/1.52476.

[21] Hawkins, M., Guo, Y., and Wie, B., "ZEM/ZEV Feedback Guidance Application to Fuel-Efficient Orbital Maneuvers Around an Irregular-Shaped Asteroid," AIAA Guidance, Navigation, and Control Conference, Minneapolis, Minnesota, USA, 13-16 August 2012, Paper AIAA 2012-5045. 